Matematisk-Naturvidenskabelig Klasse, no. 10) may be restated as follows. Given an arbitrary finite set of symbols, with $A$ 's and $B$ 's arbitrarily given strings (zeichenreihen) involving no other symbols than those in the given set, $P$ and $Q$ operational variables, to determine whether $B$ is an assertion in the system with initial assertion $A$ and operations $P A_{i} Q$ produces $P B_{i} Q, P B_{i} Q$ produces $P A_{i} Q, i=1,2, \cdots, \mu$. Through the intermediary of the Turing machine, a known recursively unsolvable decision problem is reduced to the decision problem of a system with initial assertion $A^{\prime}$ and operations $P A_{i}^{\prime} Q$ produces $P B_{i}^{\prime} Q, i=1,2, \cdots, \mu^{\prime}$, having the property that the set of assertions of the system is unchanged when the system is transformed into Thue type by adding the inverse operations $P B_{i}^{\prime} Q$ produces $P A_{i}^{\prime} Q$. The recursive unsolvability of the problem of Thue easily follows. (Received September 20,1946.)

\title{
Statistics and Probability
}

\section{H. W. Becker: Rooks and rhymes.}

Kaplansky and Riordan have shown that ${ }_{c} R_{r-1}$, or the number of ways of putting $c$ non-attacking rooks on a right-angled isosceles triangle of side $r-1$, is the Stirling number $\Delta^{r-c 0} r /(r-c) !$. This is the number of selections of $c$ points on such a chess board, such that none have any row or column index in common, an idea incidental to various statistical problems. The point sets are well ordered, in 1-to-1 correspondence with the sequations (rhyme schemes) and distribution cycles. Further classifications ${ }^{(q)} R_{r}, a R_{r}, c^{\prime} R_{r}$, and ${ }_{(c)} R_{r}$ are formulated in terms of rhyme functions according to: row location of topmost rook; number of rooks in the principal diagonal; column vacancies; and column location of the bottom rook. A typical isomorphism is ${ }_{c^{\prime}} R_{r}$ is equal to the $R_{r}$ with $c$ th column empty is equal to the number of distributions of $r+1$ men into crews, such that one man is incompatible with, and must be segregated from, $r-c$ other men. (Received July $24,1946$.

\section{Nilan Norris: An extension of an equality among averages.}

A classic theorem of algbra states that if $A, G$, and $H$ are respectively the arithmetic, geometric, and harmonic means of two positive real numbers, then $G^{2}=A H$. In this paper proof is given that a sufficient condition for the extension of this equality to any $n$ positive numbers is that the logarithms of the variates be symmetrically distributed about an axis of ordinates at $\log G$ of the $n$ numbers. For samples and populations obeying the symmetry condition with respect to $\log G$, the theorem is extended to an unlimited number of averages as yielded by certain sample and integral forms of general means (generalized means value functions). (Cf. an unpublished manuscript of J. B. Canning, A theorem concerning a certain family of averages of a certain type of frequency distribution.) (Received August 15, 1946.)

\section{TOPOLOGY}

\section{Salomon Bochner and Deane Montgomery: Groups on analytic manifolds.}

This paper studies the nature of complex and real Lie groups acting on certain complex or real manifolds in the large. For example, it proves that the group of all complex analytic homeomorphisms of a compact complex manifold is a complex Lie group. (Received September 20, 1946.) 